\title{
NutriMob Kids - Um Aplicativo Para Avaliação, Prescrição e Monitoramento da Terapia Nutricional Enteral Domiciliar em Pediatria
}

\author{
Daniel A. Callegari' ${ }^{1}$, Raquel da Luz Dias ${ }^{2}$, Ana M. P. Feoli ${ }^{2}$, \\ Murilo de O. Araújo ${ }^{1}$, Laura Hofmeister ${ }^{2}$ \\ ${ }^{1}$ Faculdade de Informática, ${ }^{2}$ Faculdade de Enfermagem, Nutrição e Fisioterapia \\ Pontifícia Universidade Católica do Rio Grande do Sul - Porto Alegre, RS - Brasil \\ \{daniel.callegari, raquel.dias, anafeoli\}@pucrs.br, \\ \{murilo.araujo, laura.hofmeister\} dacad.pucrs.br
}

\begin{abstract}
Nutritional therapy is one of the most important components of home care in pediatrics, especially the enteral modality. The use of technologies that support this kind of therapy is fundamental to the patient's quality of life. This paper describes the development of a computational solution to support the evaluation, prescription and monitoring of home enteral nutritional therapy for pediatric patients. Developed using OpenUP and supported by UML diagrams, the project's results comprise a Web site for health professionals as well as a mobile application that allows family members or caregivers to monitor guidelines for the prescribed nutritional therapy.
\end{abstract}

Resumo. Um dos principais componentes da assistência domiciliar em pediatria é a terapia nutricional enteral. O uso de tecnologias que apoiam as etapas de implementação do tratamento promove maior segurança e qualidade de vida aos profissionais, pacientes e cuidadores envolvidos. Este artigo descreve o desenvolvimento de uma solução computacional de suporte à avaliação, à prescrição e ao monitoramento da terapia nutricional enteral domiciliar para pacientes pediátricos, desenvolvido com base no OpenUP e suportado por diagramas UML. Os resultados do projeto incluem um website para profissionais de saúde e um aplicativo móvel, que permite que familiares ou cuidadores monitorem as orientações sobre a terapia nutricional prescrita.

\section{Introdução}

O perfil epidemiológico brasileiro relacionado à saúde da criança tem gerado necessidades e exigências aos serviços de saúde, tanto no que se refere a uma demanda crescente de serviços altamente especializados e complexos, quanto à necessidade de serviços básicos de qualidade e eficientes, como a estruturação da assistência domiciliar (AD) [SBP 2009]. Neste contexto, um dos importantes componentes da AD em pediatria é a terapia nutricional (TN), principalmente a terapia nutricional enteral (TNE). A TNE pode ser definida como o conjunto de procedimentos que objetiva a manutenção ou recuperação do estado nutricional do indivíduo [ANVISA 2000].

Em nível hospitalar, seu uso está diretamente relacionado com a prevenção e recuperação da desnutrição [Waitzberg et al. 2001]. Alguns pacientes já são admitidos no hospital com desnutrição e outros a desenvolvem após a internação. Já em nível 
domiciliar, a Terapia Nutricional Enteral Domiciliar (TNED) proporciona um melhor aproveitamento dos recursos disponibilizados aos cuidados assistenciais, além de permitir a humanização durante a terapia; adicionalmente, promove um ambiente familiar, confortável e seguro; reduz o tempo de internação e a exposição ao risco de infecções e, consequentemente, propicia uma melhor qualidade de vida ao paciente [Azank et al. 2009] [MS 2012].

Existem poucas evidências publicadas sobre TNE domiciliar em crianças. Um estudo realizado no Distrito Federal analisou o perfil dos pacientes pediátricos submetidos a TNED e encontrou uma casuística de 184 crianças e adolescente com idade média de 2 anos. Segundo os autores, as indicações do uso de TNED em crianças geralmente está relacionada a doenças crônicas, como os distúrbios digestivos, doenças neuromusculares, malformações congênitas e desordens cardíacas. Certamente, esse cenário está relacionado aos avanços da atenção integral à saúde da criança, o que repercute na redução das taxas de mortalidade infantil, propiciando uma maior assistência a pacientes que necessitam de cuidados crônicos [Zaban et al. 2010].

O suporte nutricional no domicílio atualmente tem propiciado o tratamento com êxito de indivíduos com enfermidades que, de outra maneira, necessitariam de internações hospitalares prolongadas ou repetidas, para a correção das alterações nutricionais. Os processos avaliativos e educativos na assistência domiciliar são fundamentais para aferir a qualidade, devendo existir parâmetros capazes de atender esse quesito [Padilha et al. 2012]. Paralelamente a esta realidade de valorização da AD e do aumento do uso de tecnologias aplicadas à saúde, observa-se um aumento crescente do uso de dispositivos móveis por indivíduos de diferentes faixas etárias e classes sociais [Biscione 2013].

\subsection{Motivação e Justificativa}

Considerando o cenário atual, o uso das TICs avançou muito nas últimas décadas no que diz respeito ao uso, à qualidade e à abrangência dos equipamentos. Os dispositivos móveis destacam-se neste sentido, pois conferem a estes equipamentos uma ampla possibilidade de customização e de usos na área da saúde. Os Personal Health Applications ou Aplicativos de Saúde Pessoal têm cada vez mais espaço nos telefones celulares e nos tablets de usuários do mundo todo. No entanto, esses dispositivos podem estar sendo subutilizados no sentido da promoção da saúde, principalmente em situações em que o monitoramento de indicadores de saúde deve fazer parte da rotina do usuário.

Atualmente existem seis aplicativos com a temática da nutrição enteral, oferecendo ao usuário soluções para cálculo de necessidades nutricionais e escolha de fórmulas; a saber: Nutricritical, NHScalc BR, Prodiet, Nutrimed, Tecnovida e Nutrimanager. Nenhum deles, contudo, possui a abrangência das funcionalidades da solução aqui proposta, que integra informações vindas do profissional de saúde e do usuário final. $\mathrm{O}$ ineditismo da proposta confere a característica inovadora desta solução. Adicionalmente, não foram encontrados outros trabalhos relacionados que se equiparem ao proposto nesta pesquisa. Dessa forma, tendo em vista a relevância do tema e a oportunidade de ampliação de uma solução computacional em pleno desenvolvimento, com uma aplicação focada na saúde da criança, foi proposto o desenvolvimento de uma solução computacional para apoiar o tratamento em TNED em pediatria. 
Este projeto é, portanto, uma continuidade do projeto de pesquisa NutriMob, que teve seu início em agosto de 2016. O objetivo inicial do projeto antecessor foi o de prototipar uma solução computacional de suporte à avaliação, à prescrição e ao monitoramento da terapia nutricional enteral domiciliar para indivíduos adultos. Tal projeto já se encontra em uma fase avançada de desenvolvimento, tanto da solução computacional do website e do aplicativo, quanto do conteúdo da solução. Devido às especificidades da TNE em pediatria - que possui avaliação e prescrição dietética distintas das do adulto - julgamos necessário o desenvolvimento de um segmento específico para pacientes pediátricos. Foram acrescentadas à solução 21 tabelas de cálculo específicas para pediatria, 7 novos gráficos e 27 novas fórmulas, além de funcionalidades exclusivas para pacientes pediátricos. Detalhes adicionais podem ser obtidos no website oficial do projeto www.nutrimob.com.br.

\subsection{Objetivos Específicos}

O objetivo principal do projeto é fornecer apoio computacional especializado tanto para os profissionais de saúde quanto para os pacientes, familiares ou cuidadores. As atividades principais do projeto são: (i) levantamento e análise das necessidades dos profissionais de saúde e dos pacientes pediátricos em TNED; (ii) elaboração de uma solução computacional para servir de apoio aos profissionais de saúde, familiares e pacientes na condução de um tratamento de nutrição enteral domiciliar; (iii) implementação de um website, por meio do qual os profissionais de saúde podem gerenciar e acompanhar os tratamentos de seus pacientes; (iv) implementação de um aplicativo móvel para ser usado pelos familiares, pelos cuidadores ou pelo próprio paciente pediátrico, por meio do qual as recomendações da terapia são comunicadas; (v) elaboração e implementação das regras de negócio baseadas nas recomendações estabelecidas por diretrizes para a terapia nutricional enteral para pacientes pediátricos; (vi) avaliação da solução com o apoio de profissionais da área da saúde.

\section{Detalhamento}

A partir do website, os profissionais da saúde podem: (i) realizar a avaliação e o acompanhamento do estado nutricional com base em indicadores antropométricos e dados subjetivos; (ii) prescrever a terapia nutricional enteral mais adequada para cada caso, estabelecendo via de acesso, método de administração, necessidades nutricionais (energia, carboidratos, proteínas, lipídios, volume de líquidos) e tipo de fórmula (o sistema auxilia todo o processo e já possui cadastradas mais de 200 fórmulas nutricionais comerciais disponíveis no mercado, com o detalhamento completo de suas características nutricionais e preparo); (iii) monitorar a evolução da terapia nutricional de cada paciente.

Para o profissional de saúde, o sistema orienta a prescrição da terapia nutricional enteral, seguindo 5 principais etapas: (i) Indicações da Terapia; (ii) Determinação das Necessidades Nutricionais, (iii) Determinação da via de acesso da Terapia, (iv) Escolha da(s) fórmula(s), e (v) Determinação do Sistema e do Método de administração. Esses recursos podem ser configurados pelo profissional que acompanha o paciente, permitindo que familiares e cuidadores ou o próprio paciente, tenham um maior conhecimento, controle e autonomia sobre o tratamento nutricional realizado no domicílio. Através do aplicativo, os usuários finais, por sua vez, podem: (i) acompanhar 
as recomendações dos profissionais da saúde; (ii) atualizar dados sobre as condutas e sobre a evolução do tratamento; (iii) receber avisos e notificações programadas, de acordo com o tratamento indicado; (iv) acompanhar orientações e intercorrências; (v) manter um registro sobre o seu tratamento. A Figura 1 apresenta alguns exemplos de telas do aplicativo desenvolvido para a plataforma Android. As principais linguagens e tecnologias utilizadas foram: Java, Javascript, PHP, ChartJS, MySQL; bem como Selenium IDE e Postman para a execução de testes.

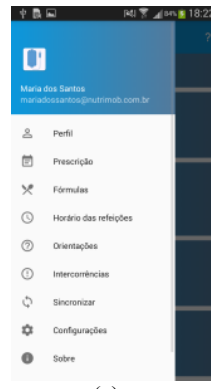

(a)

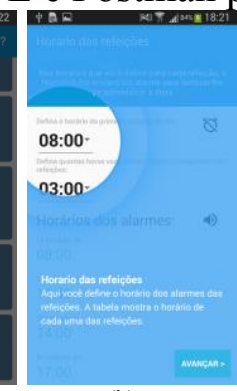

(b)

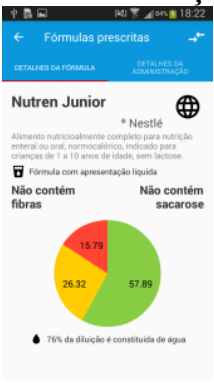

(c)

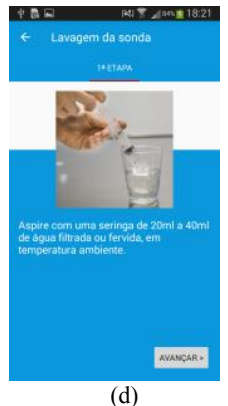

(d) (a) menu de funcionalidades disponíveis;

(b) exemplo do assistente de configuração do aplicativo, o qual é executado automaticamente no primeiro uso;

(c) detalhamento de uma das fórmulas nutricionais prescritas ao paciente;

(d) exemplo de etapa disponível na seção de orientações ao paciente (fotografias produzidas pela própria equipe em ambiente especializado).

Figura 1. Exemplos de telas do aplicativo

\section{Conclusão e Trabalhos Futuros}

Como produto deste projeto e principal resultado está o desenvolvimento de uma solução computacional para apoiar o tratamento em Terapia Nutricional Enteral Domiciliar em pediatria, que promoverá a maior segurança nas decisões clínicas dos profissionais e maior conhecimento sobre o tratamento e autonomia para o autocuidado nos pacientes e seus cuidadores. As atividades futuras já agendadas envolvem a avaliação da usabilidade com usuários finais e profissionais parceiros.

\section{Referências}

ANVISA. (2000) Agência Nacional de Vigilância Sanitária. "Resolução RDC nº 63 de 06/07/2000". Aprova o Regulamento Técnico para fixar os requisitos mínimos exigidos para a Terapia de Nutrição Enteral. Brasília, DF.

Azank, A.T.; Leandro-Merhi, V.A.; Poliselli, C.; Oliveira MRM. (2009) "Indicadores nutricionais em pacientes alimentados por sonda, em sistema de "Home Care", Arquivos Catarinenses de Medicina Vol. 38, no. 4.

Biscione F.M, et.al. Avaliação de efetividade da atenção domiciliar de uma cooperativa médica de Belo Horizonte, MG, Cad. Saúde Pública, RJ 29 Sup: S73-S80, 2013. 9.

MS - Ministério da Saúde. (2012) Caderno de Atenção Domiciliar. Brasília.

Padilha, P.C.; Souza, T.V.; Botelho, A.; Buonora, S. (2012) "Assistência Domiciliar em Pediatria: Enfoque na Terapia Nutricional Enteral”, Boletim da Sociedade Brasileira de Nutrição Enteral e Parenteral - SBNPE. Rio de Janeiro, Jul/Ago/Set.

SBP - Sociedade Brasileira de Pediatria. (2009) "Avaliação nutricional da criança e do adolescente" - Manual de Orientação / Sociedade Brasileira de Pediatria. Departamento de Nutrologia, $112 \mathrm{p}$.

Waitzberg, D.L.; Caiaffa, W.T.; Correia, M.I. (2001) "Hospital malnutrition: the Brazilian national survey (IBRANUTRI): a study of 4000 patients", Nutrition. Volume 17, Issues 7-8, July-August, Pages 573-580.

Zaban, A.L.R.; Novaes, M.R.C.G. (2010) "Home enteral nutrition in children: a oneyear experience with 184 patients in Distrito Federal, Brazil”. Experimental Biology and Medicine; 235:584-9. 